\title{
ON THE INTERIOR OF THE CONVEX HULL OF A EUCLIDEAN SET
}

\author{
WILLIAM GUSTIN
}

In this note we shall prove for each positive integer $n$ the following theorem $\Delta_{n}$ concerning convex sets in an $n$-dimensional euclidean space.

Theorem $\Delta_{n}$. Any point interior to the convex hull of a set $E$ in an n-dimensional euclidean space is interior to the convex hull of some subset of $E$ containing at most $2 n$ points.

This theorem is similar to the well known result that any point in the convex hull of a set $E$ in an $n$-dimensional euclidean space lies in the convex hull of some subset of $E$ containing at most $n+1$ points $[1,2] .{ }^{1}$ In these theorems the set $E$ is an arbitrary set in the space. The convex hull of $E$, denoted by $H(E)$, is the set product of all convex sets in the space which contain $E$.

A euclidean subspace of dimension $n-1$ in an $n$-dimensional euclidean space will be called a plane. Every plane in an $n$-dimensional euclidean space separates its complement in the space into two convex open sets, called open half-spaces, whose closures are convex closed sets, called closed half-spaces. If each of the two open halfspaces bounded by a plane $L$ intersects a given set $E$, then $L$ is said to be a separating plane of $E$; otherwise $L$ is said to be a nonseparating plane of $E$.

In order to prove our sequence of theorems we shall make use of the following result: A point $i$ is interior to the convex hull of a set $E$ in an $n$-dimensional euclidean space if and only if every plane through $i$ is a separating plane of $E[1]$.

We prove our sequence of theorems by induction. The proof of Theorem $\Delta_{1}$ is trivial and will be omitted. Now suppose that Theorem $\Delta_{n-1}$ is true for an integer $n>1$. We shall show that Theorem $\Delta_{n}$ is also true. To this end let $i$ be a point interior to the convex hull of a set $E$ in an $n$-dimensional euclidean space. We are to demonstrate that $i$ is interior to the convex hull of some subset $P$ of $E$ containing at most $2 n$ points.

First we show that $i$ is interior to the convex hull of some finite subset $Q$ of $E$. Since $i$ is interior to $H(E)$, it is interior to a simplex 1946.

Received by the editors September 19, 1946, and, in revised form, November 16,

${ }^{1}$ Numbers in brackets refer to the references cited at the end of the paper. 
lying in $H(E)$. Consider the $n+1$ vertices $q_{k}(k=1, \cdots, n+1)$ of such a simplex. The vertex $q_{k}$ lies in $H(E)$ and hence, according to the previously mentioned result of Carathéodory and Steinitz, lies in the convex hull of some subset $Q_{k}$ of $E$ containing at most $n+1$ points. The set $Q=\sum_{k} Q_{k}$ is then a finite subset of $E$ containing at most $(n+1)^{2}$ points. Evidently the convex hull of this set contains the simplex with vertices $q_{k}$ and hence contains the point $i$ in its interior. ${ }^{2}$

Since $Q$ is finite, there exists a subset $P$ of $Q$ which contains the point $i$ in the interior of its convex hull and which is irreducible with respect to this property. Let $p$ be a definite point of $P$. Then $i$ is not an interior point of $H(P-p)$, so some plane $L$ through $i$ is a nonseparating plane of $P-p$. Let $D$ be that one of the two open halfspaces bounded by $L$ which is disjoint with $P-p$ and let $D^{\prime}$ be the other open half-space. Thus $P-p$ lies in the closed half-space $\bar{D}^{\prime}$ complementary to $D$.

Since $i$ is an interior point of the convex hull of $P$, the open halfspace $D$ contains a point of $P$. This point must be $p$, for $D$ contains no point of $P-p$. Similarly the open half-space $D^{\prime}$ contains a point $p^{\prime}$ of $P$. We shall use this point $p^{\prime}$ later in the proof.

Consider an arbitrary point $x$ of the closed half-space $\bar{D}^{\prime}$. Since $p$ lies in the complementary open half-space $D$, the line segment $H(p+x)$ intersects the boundary $L$ of $D$ in exactly one point which we denote by $\phi(x)$. Thus $\phi(x)$ is the projection of $x$ from $p$ onto $L$.

The projection $\phi$ is $1-1$ over the subset $P-p$ of the closed half-space $\bar{D}^{\prime}$. For suppose, to the contrary, that some two points $p_{1}$ and $p_{2}$ of $P-p$ project into the same point of $L$. The three points $p, p_{1}$, and $p_{2}$ are then collinear. Now $p$ does not lie between the other two points, else the open half-space $D$ containing $p$ would contain at least one of these other two points. We may then assume $p_{1}$ and $p_{2}$ to be so labeled that a linear order of the three points is $p_{,} p_{1}, p_{2}$. Therefore

$$
p_{1} \subset H\left(p+p_{2}\right) \subset H\left(P-p_{1}\right),
$$

so the sets $H\left(P-p_{1}\right)$ and $H(P)$ are identical. The point $i$ is then interior to $H\left(P-p_{1}\right)$ in contradiction to the irreducibility of $P$.

The projection of the convex hull of a set is the convex hull of the projection of that set, and the projection of an interior point of a convex set is an interior point of the projection of that set [3]. Therefore the point $\phi(i)=i$ is an interior point of the set $\phi(H(P-p))$ $=H(\phi(P-p))$ in the euclidean subspace $L$ of dimension $n-1$. Ac-

2 That $i$ is interior to the convex hull of some finite subset of $E$ may also be proved by the Heine-Borel theorem. I am indebted to the referee for the above proof. 
cording to Theorem $\Delta_{n-1}$ the point $i$ is an interior point in $L$ of the convex hull of some subset $P_{L}$ of $\phi(P-p)$ containing at most $2 n-2$ points. Define

$$
P^{*}=p+P \phi^{-1}\left(P_{L}\right)+p^{\prime} \text {. }
$$

Since the projection $\phi$ is $1-1$ over $P-p$, the set $P \phi^{-1}\left(P_{L}\right)$ is a subset of $P$ containing at most $2 n-2$ points. Therefore $P^{*}$ is a subset of $P$ containing at most $2 n$ points.

We shall show that $i$ is interior to $H\left(P^{*}\right)$. First we notice that the coplanar set $P_{L}$ lies in $H\left(P^{*}\right)$. For, if $x$ is an arbitrary point of $P \phi^{-1}\left(P_{L}\right)$, then

$$
\phi(x) \subset H(p+x) \subset H\left(P^{*}\right),
$$

since both $p$ and $x$ lie in $H\left(P^{*}\right)$. Now consider the pyramid $H\left(p+P_{L}\right)$ whose apex $p$ lies in $D$ and whose base $H\left(P_{L}\right)$ lies in $L$. The point $i$ is an interior point in $L$ of the base $H\left(P_{L}\right)$ of this pyramid, so some closed hemisphere $A$ with center $i$ and base on $L$ lies in $H\left(p+P_{L}\right)$. Similarly, some closed hemisphere $A^{\prime}$ with center $i$ and base on $L$ lies in the pyramid $H\left(p^{\prime}+P_{L}\right)$. Evidently there exists a sphere $I$ with center $i$ such that $I \subset A+A^{\prime} \subset H\left(p+P_{L}\right)+H\left(p^{\prime}+P_{L}\right) \subset H\left(P^{*}\right)$. The point $i$ is then interior to the convex hull of the subset $P^{*}$ of $P$. From the irreducibility of $P$ it follows that $P^{*}=P$. Therefore $P$ contains at most $2 n$ points.

Thus for every integer $n>1$, Theorem $\Delta_{n-1}$ implies Theorem $\Delta_{n}$. Since Theorem $\Delta_{1}$ is true, we conclude by induction that Theorem $\Delta_{n}$ is true for each positive integer $n$.

The following example shows that the number $2 n$ in Theorem $\Delta_{n}$ cannot be improved. Let $i$ be the zero point of an $n$-dimensional vector space. Choose any $n$ linearly independent and hence nonzero points in this space. Let $E$ be the set consisting of these points and their vector negatives; $E$ then contains $2 n$ points. It is easy to show that the zero point $i$ is interior to the convex hull of $E$ but is not interior to the convex hull of any proper subset of $E$.

\section{REFERENCES}

1. C. Carathéodory, Über den Variabilitatsbereich der Fourier'schen Konstanten von positiven harmonischen Funktionen, Rend. Circ. Mat. Palermo vol. 32 (1911) \$9.

2. E. Steinitz, Bedingt konvergente Reihen und konvexe Systeme, J. Reine Angew. Math. vol. 143 (1913) \$10.

3. - - Bedingt konvergente Reihen und konvexe Systeme, J. Reine Angew. Math. vol. 146 (1916) §26.

University of California at Los ANGeles 